\title{
MIGRATIONS VERTICALES : QUELQUES DONNEES SUR LE COMPORTMENT AUTOMNAL DU ZOOPLANCTON D'UN CANAL DE NAVIGATION
}

\author{
par Nadine ANGELI'1.
}

Les migrations verticales de 9 espèces de Métażoaires (Crustacés et Rotifères) ont été observées en novembre dans un canal dont la profondeur maximale ne dépasse pas $2,60 \mathrm{~m}$.

Les récoltes furent effectuées toutes les trois heures à trois niveaux différents, durant une période de 24 heures.

Brachionus calyciflorus, $B$, angularis, Polyarthra vulgaris, Filinia longiseta et Bosmina longirostris effectuent trois mouvements ascendants successifs qui surviennent respectivement avant et après l'aurore puis au crépuscule.

Une migration inversée a été observée pour Keratella quadrata (dont la densité maximale fut atteinte à proximité du fond vers 23 heures) et pour les nauplii de Cyclopidae qui migrent massivement vers la surface durant la période de plus forte illumination.

La distribution verticale du plancton était à peu près uniforme entre 23 heures et 2 heures du matin.

\section{Vertical migrations : some data on the autumnal behaviour of zooplankton in a canal.}

In November, the vertical migrations of nine planktonic metazoa (Crustacea and Rotifers) were observed in a canal of $2.60 \mathrm{~m}$ maximal depth.

Sampling was performed every three hours at three distinct levels over a period of 24 hours.

Three successive ascendant movements occuring before and after dawn and close to twilight were shown by Brachionus calyciflorus, B. angularis, Polyarthra vulgaris, Filinia longiseta and Bosmina longirostris.

A reversed migration was observed for two species : after sunset for Keratella quadrata, whose maximal density occured near to the bottom at $23.00 \mathrm{~h}$ and close to noon for the nauplii of Cyclopidae.

From 23.00-02.00 h, the vertical distribution of the different species was almost uniform.

\section{INTRODUCTION}

Depuis la fin du siècle dernier, les migrations de plancton ont fait l'objet d'observations relativement nombrcuses. Les travaux axés sur ces phénomènes présentent deux points communs : ils

1. Laboratoire de Protistologie et Microscopie électronique, Université des Sciences et Techniques de Lille I, B. P. 36, 59650 Villeneuve-d'Ascq.

Ce travail a bénéficié de l'aide matérielle du C.N.R.S., F.R.A., n ${ }^{\circ} 184$. 
portent, dans la très grande majorité des cas, sur des eaux limpides et profondes (lacs de montagne ou domaine océanique) et, à l'exception de quelques travaux comme ceux de George et al. (1969), Schindler et al. (1971) ou de Larsson (1970), les observations sont les plus souvent limitées à la belle saison.

Dans ce domaine d'études, les pelites collections d'eau aux profondeurs modestes font figure de parent pauvre et les migrations moins spectaculaires dans ces dernières n'ont retenu l'attention que d'un nombre restreint de Limnologistes.

Cette étude préliminaire effectuée sur un canal a été motivée par différentes raisons : tout d'abord, des observations étant entreprises sur le profil biologique saisonnier de ce canal, il était souhaitable de disposer d'informations complémentaires sur le comportement des espèces dominantes. En outre, pour des questions pratiques d'échantillonnages, il importait de savoir s'il se produit ou non des migrations et si oui, de connaître leur ampleur. Enfin, vu la relative hétérogénéité diurne du plancton, il était intéressant de savoir si les organismes étaient susceptibles de présenter une distribution normale, à un moment donné du cycle nycthéméral, comme le laissent supposer les travaux de Hall et al. (1970).

Cette étude a été effectuée en novembre. Le choix de la saison froide se justifiait par le manque de données relatives à l'automne et l'hiver.

\section{I. - MATÉrIEL ET MẼTHODES}

Le canal de Seclin constitue une voie navigable très modeste $(6 \mathrm{~km})$ et pratiquement inutilisée depuis quelques années. Il relie l'agglomération de Seclin au canal très pollué de la Deule.

Les prélèvements ont été effectués en amont à $500 \mathrm{~m} \mathrm{du}$ 《port » de Seclin, dans un secteur qui échappe totalement à la pollution consécutive à la pénétration des eaux de la Deule (Angeli 1972). Il s'y développe un plancton abondant et relativement varié, caractérisé par des espèces communes aux milieux eutrophes calciques.

A l'époque des prélèvements (novembre 1969), l'amont du canal comptait encore de nombreux îlots de phanérogames aquatiques, constitués principalement par Nuphar lutea et qui s'épanouissaient librement dans le chenal; dans la zone littorale par contre, les travaux d'entretien des berges interdisaient tout développement de la végétation.

Afin que la distribution du plancton au cours du cycle nycthéméral, ne soit pas perturbée par la proximité de ces herbiers, les récoltes ont été faites dans une zone totalcment dépourvue de macrophytes. Cette distribution durant la période diurne est assez hétérogène. Les organismes se répartissent selon deux gradients : 
un gradient horizontal (l'influence de la zone littorale se fait sentir à plusieurs mètres des berges alors que la pleine eau proprement dite est limitée à un étroit chenal médiant), un gradient vertical (en liaison avec la distribution de la nourriture disponible et la pénétration de la lumière).

Les échantillons quantitatifs ont été récoltés à l'aide d'un flacon lesté d'une capacité de deux litres : modèle classique de Meyer dont le bouchon solidaire de la corde est attaché par une entrave plus courte que celle du flacon. L'ajustage est fait de telle façon qu'une fois la corde tendue, une légère secousse suffit à déboucher le goulot. Des repères portés sur la corde de $10 \mathrm{~cm}$ en $10 \mathrm{~cm}$ permettent d'effectuer les prélèvements aux profondeurs désirées. Ce matériel rustique donne des résultats tout à fait acceptables pourvu que les profondeurs choisies diffèrent de plusicurs dizaines de $\mathrm{cm}$.

En l'occurrence, les récoltes ont été réalisées à proximité de la surface $(-0,20 \mathrm{~m})$, légèrement en dessous de la limite de visibilité du disque de Secchi $(1,20 \mathrm{~m})$ et à proximité du fond $(-2,50 \mathrm{~m})$.

Pour chaque profondeur, un échantillon moyen a été constitué à partir de 5 prélèvements ponctuels, effectués de $2 \mathrm{~m}$ en $2 \mathrm{~m}$ dans la partie médiane du chenal. Trois séries d'échantillons complétées par une prise au filet en surface ont ainsi été récoltécs toutes les trois heures, 24 heures durant.

Le matériel filtré sur de la soie à bluter (vide de maille de $30 \mu$ ) a été fixé par un apport de $5 \%$ de formol neutralisé.

Les récoltes ont été effectuées durant la nouvelle lune et en absence de toute turbulence atmosphérique. La transparence en cette saison ne dépassait pas $1 \mathrm{~m}$ (mesure au Secchi).

Trois facteurs ont été enregistrés : la température, le $\mathrm{pH}$ (à l'aide d'un pH mètre portatif), et l'oxygène dissous (méthode de Winkler modifiée).

Les comptages ont été faits au microscope à inversion dans des chambres à décantation avec un grossissement de 120 fois.

\section{2. - RÉSULTATS}

\section{Données physico-chimiques.}

Les variations mesurées sont insignifiantes pour le $\mathrm{pH}$, et relativement faibles pour la température et l'oxygène dissous dont les écarts maxima respectifs sont en surface de $4,5{ }^{\circ} \mathrm{C}$ et de $2 \mathrm{mg} / \mathrm{l}$ d'oxygène (fig. 1). 


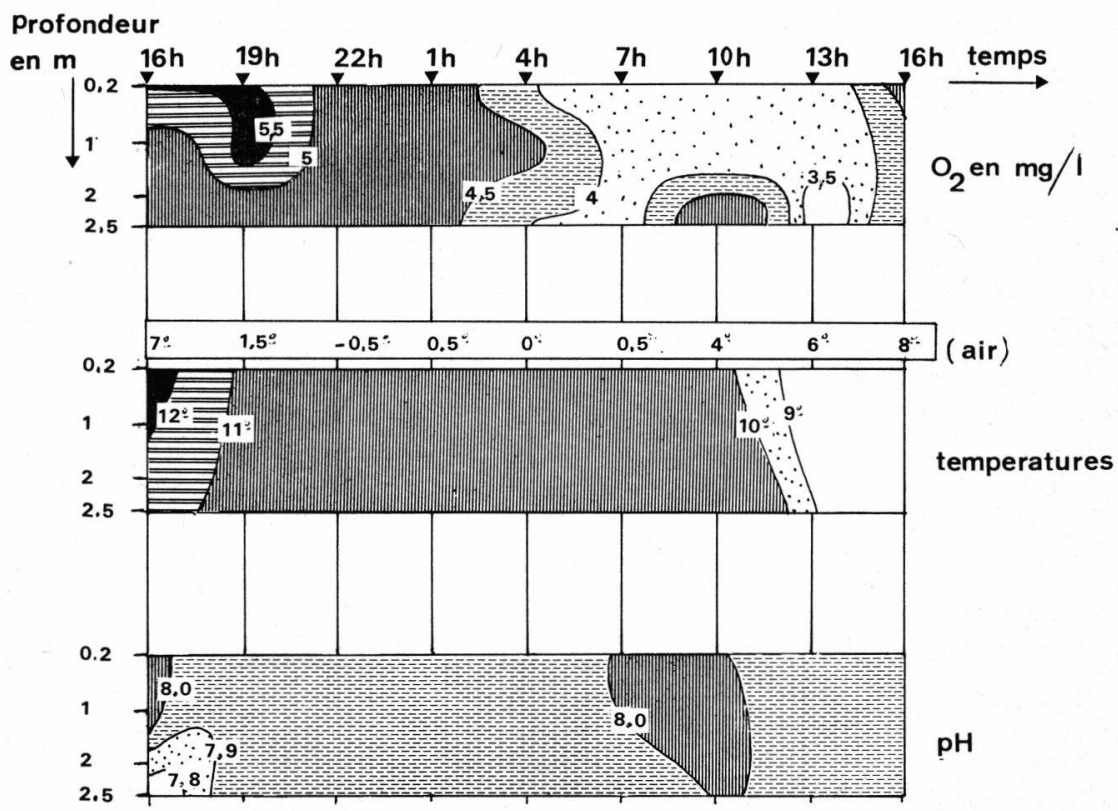

Fig. 1. - Évolution de l'oxygène dissous, de la température et du $\mathrm{pH}$ au cours des récoltes.

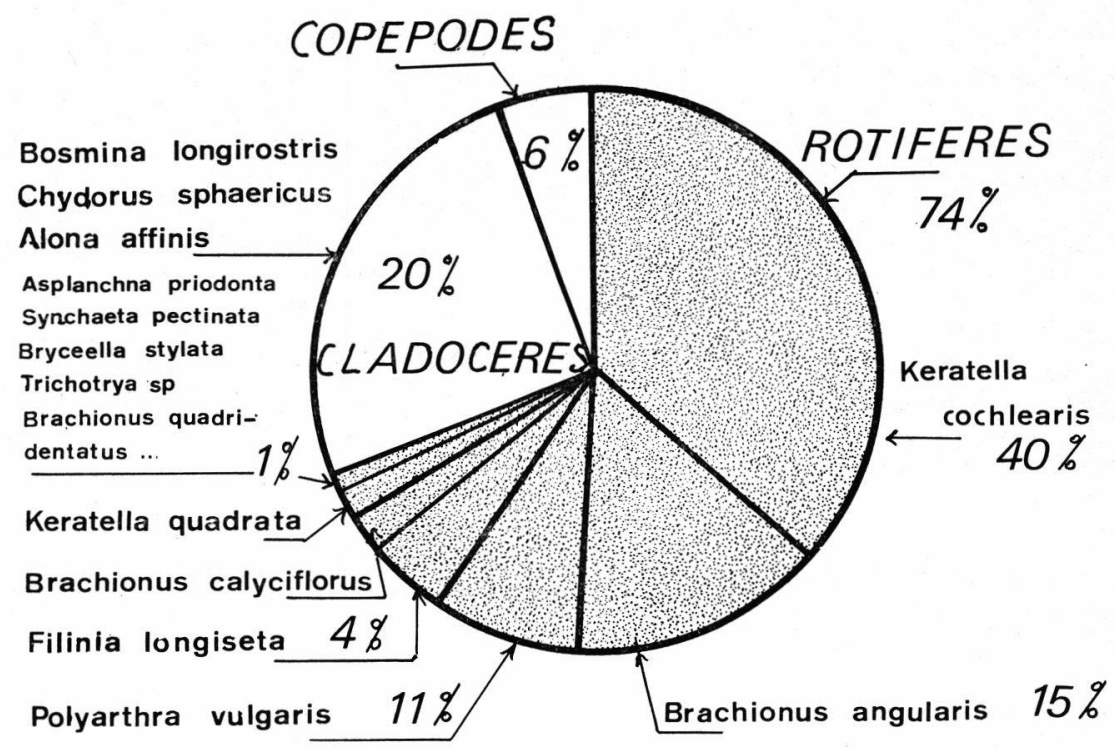

Fig. 2. - Composition qualitative du zooplancton. 


\section{Données biologiques.}

\section{a. Espèces non dominantes.}

Quelques Cladocères appartenant à des espèces typiquement benthiques ont été trouvées en surface dans les échantillons récoltés au filet. Ces espèces : Rhynchotalona rostrata (Koch), Graptoleberis testudinaria (Fischer) et Ilyocryptus sp. sont apparues de façon sporadique dans le plancton de surface de 22 heures à 4 heures.

Deux espèces moins strictement inféodées à la zone benthique (Chydorus sphaericus (O. F. Müller), Alona affinis (Leydig), très peu représentées dans les récoltes diurnes, apparaissaient en plus grande abondance dans les prises nocturnes au filet.

\section{b. Espèces dominantes et adominantes.}

A partir d'une population dont la composition globale est donnée par le graphique 2, les fluctuations de densités observées de 3 heures en 3 heures au cours des 24 heures sont illustrées dans les graphiques 3 à 5 .

Sur les figures 3 et 4 , les densités par litre ont été reportées sur chacun des axes correspondant aux différentes heures de prélèvements. Les courbes obtenues réunissent les points isobathes et permettent de suivre les fluctuations qui se manifestent à chacun des niveaux considérés.

Exprimés sous cette forme, les résultats ne rendent pas compte des seules migrations verticales. En effet, ces variations de densité en une même profondeur peuvent également résulter de mouvements horizontaux. On constate ainsi qu'au cours des 24 heures, la somme des densités relatives aux trois profondeurs passe par un maximum vers 10 heures et un minimum vers 1 heure.

La surabondance des organismes, vers 10 heures dans les 3 séries d'échantillons, est particulièrement spectaculaire pour le genre Filinia.

Pour pallier l'interférence des mouvements horizontaux, un autre mode d'expression a été utilisé (fig. 5). Chaque valeur est exprimée en pourcentage de la somme des densités de la série correspondante.

\section{3. - INTERPRÉTATION DES DONNÉES}

\section{Mouvements des espèces présentant un phototropisme négatif aux fortes luminosités.}

Les Rotifères et Cladocères effectuent une migration diurne de forte amplitude caractérisée par l'augmentation de la densité des organismes en surface, 2 heures après le lever du soleil. Durant 


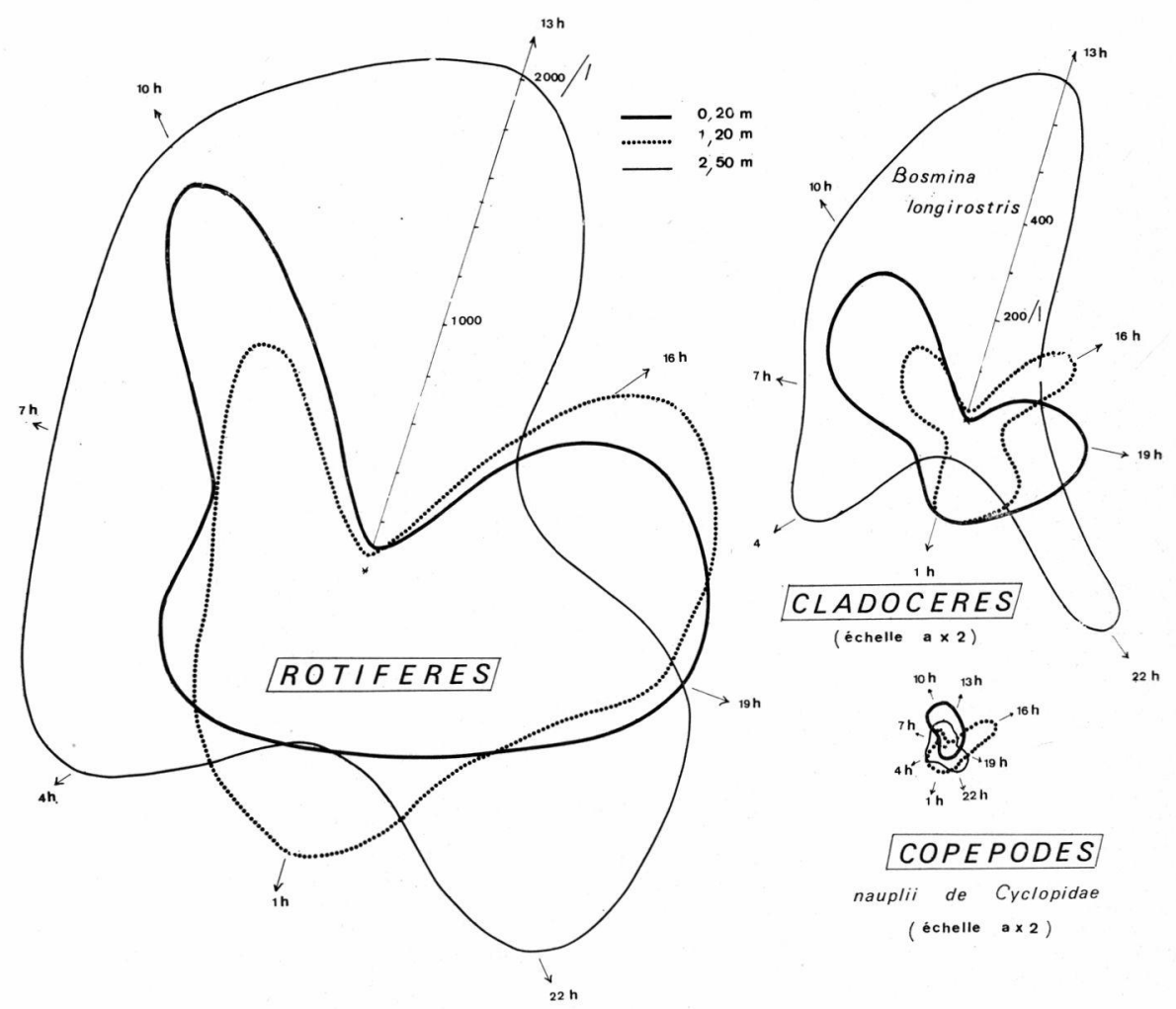

Fig. 3. - Fluctuations de densités enregistrées au cours d'un cycle de 24 heures. Les densités sont reportées à partir du centre sur chacun des axes correspondant aux 8 séries de prélèvements.

les heures de forte luminosité, les couches superficielles et miprofondes sont totalement désertées par ces organismes qui gagent à nouveau la surface au crépuscule.

Ces fluctuations de la densité dans les strates superficielles correspondent à un mouvement ascendant qui prend naissance au lever du jour et culmine vers 10 heures. Les organismes parvenus en surface ou à mi-profondeur, vont ensuite amorcer un mouvement descendant qui culmine vers 13 heures. L'amplitude de ce mouvement est telle que, durant quelques heures, on ne retrouve plus guère de Rotifères ou de Cladocères dans la moitié supérieure de la colonne d'eau (exception faite de Keratella quadrata qui présente alors un maximum à $1,20 \mathrm{~m}$ de profondeur). Lorsque la luminosité diminue, une partie de la population amorce un nouveau mouvement ascendant qui s'achève selon les espèces entre 16 et 19 heures. 

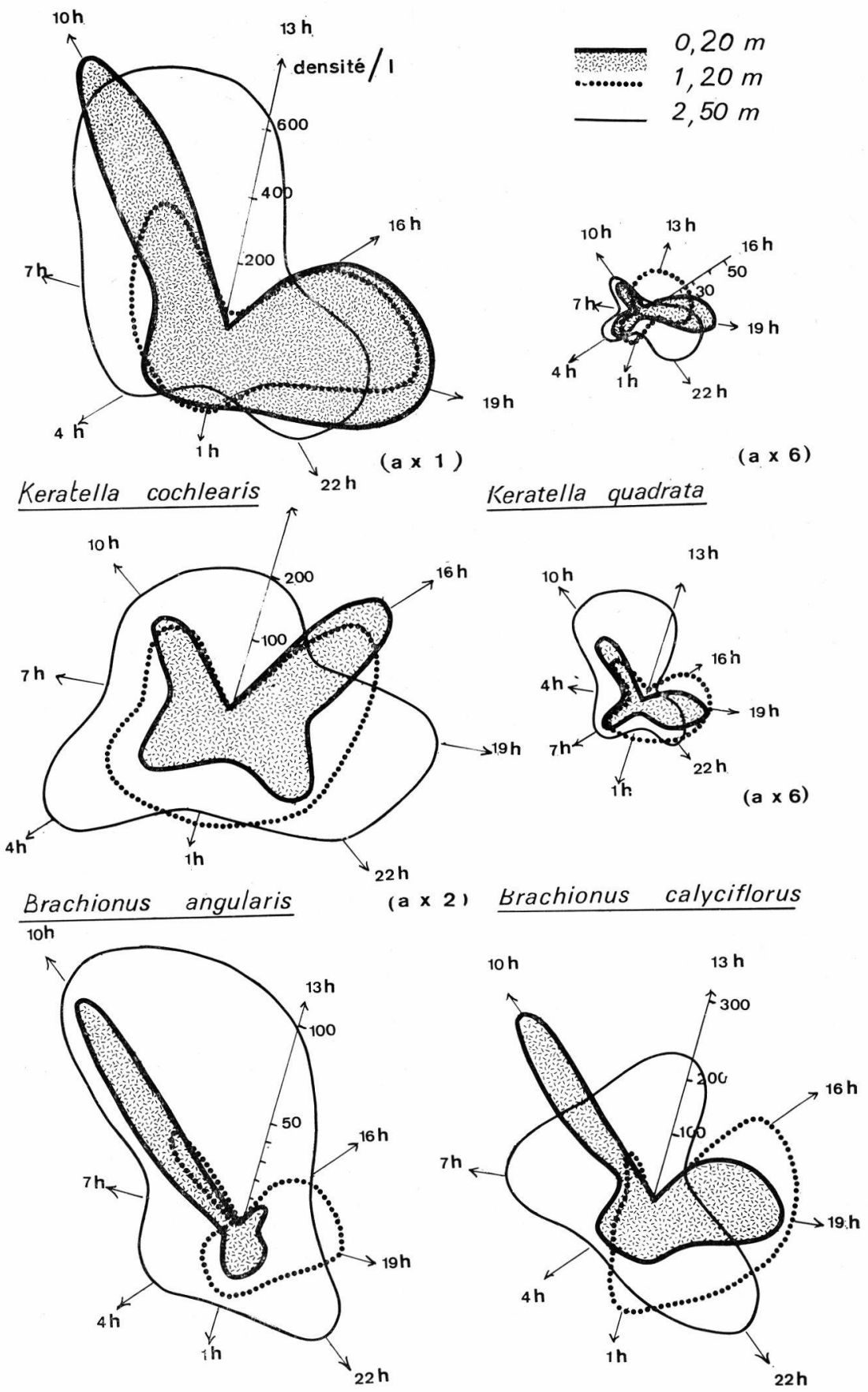

\section{Filinia longiseta}

(a $\times 6)$ Polyarthra vulgaris $(a \times 2)$

Fig. 4. - Fluctuations de densités enregistrées en différentes profonceurs au cours d'un cycle nycthéméral (plancton rotiférien). Les densités sont reportées à partir du centre sur chacun des axes correspondant aux 8 séries de prélèvements. 
Cette montée crépusculaire précoce est sans doute liée au fait qu'en novembre la luminosité est considérablement réduite dès 15 heures.

Les espèces les plus tolérantes vis-à-vis de la lumière achèvent très tôt cette migration ascendante ( 16 heures pour Filinia longiseta et Brachionus angularis) alors que les espèces apparemment plus sensibles aux radiations lumineuses (Keratella cochlearis, Brachionus calyciflorus, Polyarthra vulgaris et Bosmina longisrostris) accusent à cette heure un maximum à mi-profondeur, et ne présentent leur maximum de densité en surface que vers 19 heures. La migration ascendante de $K$. quadrata amorcée précocement se poursuit également jusqu’à 19 heures (fig. 5).
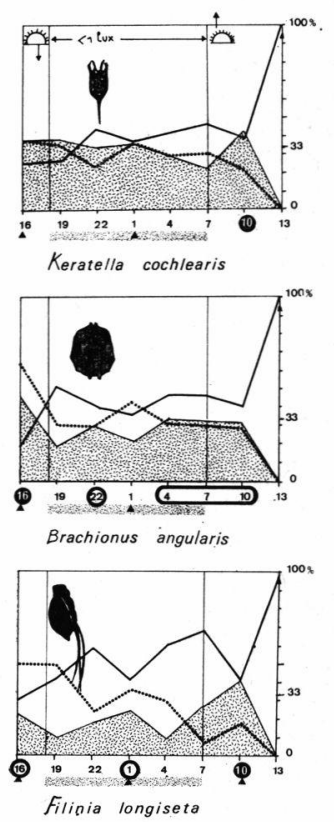

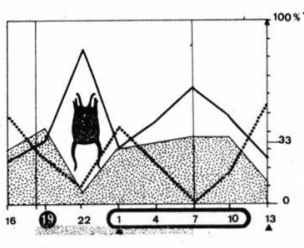

Keratella quadrata

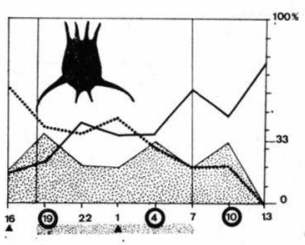

Brachionus calyciflorus

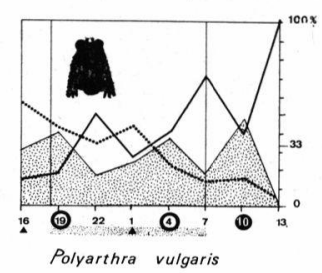

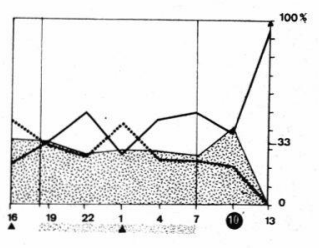

faunule rotiferienne (totale)

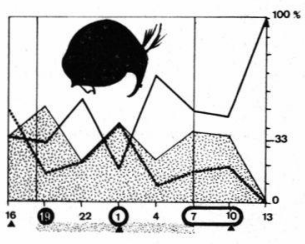

Bosmina longirostris

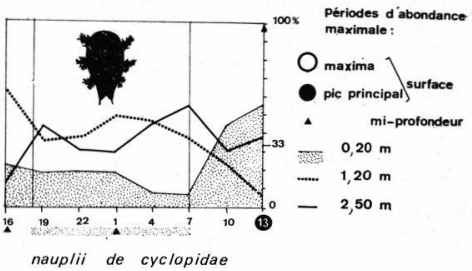

Fig. 5. - Fluctuations de la densité des espèces dominantes en surface, à miprofondeur, et au voisinage $\mathrm{du}$ fond, au cours du cycle nycthéméral. Pour chacune des séries de récoltes, les valeurs sont exprimées en \% de la somme des 3 densités correspondantes.

Durant la nuit, on constate une troisième augmentation de la densité des organismes en surface. Ce pic est plus ou moins accusé selon les espèces et coïncide avec la moitié de la nuit pour Filinia longiseta et Bosmina longirostris, alors qu'il apparaît plus tard (un peu avant l'aube) pour Brachionus calyciflorus et Polyarthra vulgaris. Ce mouvement ascendant est légèrement plus précoce 
chez Brachionus angularis. On constate ensuite un dernier mouvement descendant nocturne qui s'amorce peu avant l'aurore.

\section{Migrations particulières des nauplii.}

Le matériel utilisé pour les récoltes ne permet pas d'obtenir des échantillons représentatifs pour les Cyclopidae adultes. Ceux-ci, par leur nage vigoureuse, fuient les turbulences créées par l'emplissage du flacon lesté. Nous ne possédons des données quantitatives que pour les stades copépodites (trop peu abondants pour que l'on puisse interpréter leurs fluctuations de densité) et les nauplii. Ces derniers ont un comportement tout à fait différent de celui des autres organismes : en surface leur densité maximale est atteinte durant la période de plus forte luminosité. Cette migration diurne inversée touche une partie importante de la population, puisque le taux d'organismes en surface atteint $50 \%$ de la somme des densités observées aux 3 profondeurs (fig. 5). Quelques heures plus tard, les nauplii ayant amorcé un rapide mouvement descendant dès le déclin de la luminosité, c'est dans la couche médiane que se trouve concentrée la majorité des individus.

Contrairement aux Cladocères et aux Rotifères, chez ces jeunes Cyclopidae, la majeure partie de la population se cantonne dans les couches moyennes et protondes durant la nuit.

\section{Amplitudes de ces migrations.}

- Les migrations diurnes.

Pour les Rotifères et les Bosmines, le mouvement descendant diurne est très fortement marqué et affecte la quasi totalité de la population qui, à l'exception des individus de Keratella quadrata (concentrés dans les strates moyennes), déserte totalement la surface et les couches moyennes pour se concentrer près du fond.

Chez les nauplii, le mouvement ascendant qui prend place en cette période est également de grande amplitude.

- Les migrations nocturnes.

Des migrations de forte amplitude sont constatées chez Bosmina longirostris et Keratella quadrata. Cette dernière, contrairement aux autres espèces présente des migrations de plus grande amplitude la nuit que le jour.

\section{4. - DISCUSSION}

En nous reportant à la littérature, nous sommes amenés à confronter ces observations avec celles d'auteurs ayant étudié, en 
une saison différente, des écosystèmes présentant fort peu de points communs avec ce canal.

Toutefois, étant donné que les variations d'intensité lumineuse constituent un facteur déterminant des migrations diurnes (en liaison avec la température et le temps), on peut se permettre de rapprocher les phénomènes observés dans la zone photique d'un lac de ceux observés dans un étang ou un canal. En novembre, la turbidité du canal de Seclin était assez élevée et la zone photique atteignait à peine $2,50 \mathrm{~m}$. C'est avant tout l'échelle à laquelle se préduisent les phénomènes qui est différente : à peine $2,50 \mathrm{~m}$ dans le canal de Seclin contre 15 à 40 mètres en eau moins riche.

\section{Rotifères et Cladocères.}

En novembre, les mouvements observés dans le canal de Seclin pour les Rotifères et les Cladocères, se rapprochent de la migration «crépusculaire» typique décrite par Cushing (1951) puis Siebeck (1960) (Twilight migrations), caractérisée par des mouvements ascendants aux périodes de faible illumination (aube et crépuscule) et un mouvement descendant très accusé durant la période de forte illumination (milieu du jour), moins marqué durant la période nocturne (« diurnal sinking» survenant vers la moitié de la nuit).

Le mouvement ascendant crépusculaire culmine selon les espèces et le biotope entre le crépuscule et la moitié de la nuit. Ainsi en été, Worthington (1931) observe vers minuit l'apparition massive en surface de tous les Cladocères, Schröder (1959) fait état d'un maximum nocturne beaucoup plus précoce $(20 \mathrm{~h})$, soit 2 heures après le coucher du soleil. Dans le canal de Seclin, nous avons vu que ce pic post-crépusculaire est très net, mais survient beaucoup plus tôt (il culmine au coucher du soleil pour Keratella cochlearis, Filinia longiseta et Brachionus angularis, environ 3 heures plus tard pour Bosmina longirostris, Keratella quadrata. Cette précocité pourrait être liée au fait qu'à l'époque de nos récoltes, la durée d'ensoleillement est plus courte qu'à la belle saison. Pour le plancton rotiférien, Sládeček, 1959, relate la concentration à quelques mètres de la surface de Keratella cochlearis à 1 heure alors que Polyarthra sp. n'achève sa migration qu'à l'aube. Dans le canal, les individus de Keratella cochlearis ayant migré vers la surface au crépuscule, y demeurent jusqu'à l'aube. Par contre, Polyarthra vulgaris présente deux maxima nocturnes d'égale intensité : l'un au crépuscule, l'autre peu avant l'aube.

En ce qui concerne les migrations diurnes des Rotifères de ce canal, les maxima enregistrés pour toutes les espèces vers 10 heures puis de 16 à 19 heures rappellent en partie les phénomènes observés par Pawłowski et George. Pawłowski (1970) constatait en juillet, dans un bras mort de la rivière Grabia que la concentration 
la plus élevée en Rotifères était atteinte en surface vers 11 heures puis vers 1 heure. Georges (1960) étudiant deux petits étangs tropicaux, observait dans l'un, où prédominaient les Rotifères Brachionus caudatus et $B$. diversicornis, un maximum de densité en surface dès 16 heures (soit en région tropicale : approximativement 2 heures avant la tombée de la nuit), alors que dans l'autre étang, où prédominaient Filinia longiseta, Keratella valga et Brachionus calyciflorus, les Rotifères présentaient un maximum vers 10 heures. Dans le canal de Seclin, pour Filinia longiseta et Polyarthra vulgaris, c'est le maximum de $\mathbf{1 0}$ heures qui est le plus accusé, alors que les espèces du genre Brachionus offrent des maxima nocturnes et diurnes de même amplitude.

En dehors des périodes de forte luminosité, il est curieux de constater que pour Bosmina longirostris, la densité observée dans les couches superficielles est toujours nettement plus importante que celles des couches moyennes du canal. Il semble qu'un bon tiers de la population présente une affinité particulière pour la surface, alors que la majorité des individus préfère évoluer en eau profonde. Tout se passe comme si l'on avait affaire à deux populations distinctes : l'une inféodée au benthos et peu sensible aux radiations lumineuses, l'autre préférant les couches superficielles, mais influencée par les fluctuations de luminosité.

En fait, si Bosmina longirostris colonise généralement les couches superficielles des lacs (Pennak 1944; Omaly 1970) et présente selon ces auteurs de très faibles migrations, il peut advenir que certaines souches se comportent différemment. Sládeček (1959), dans un lac de barrage, constate que cette espèce se cantonne dans l'hypolimnion, dans une zone benthique de quelques mètres d'épaisseur, alors que Kikuchi (1930) relate pour un lac japonais, un troisième type de comportement : $B$. longirostris étant strictement limitée dans sa distribution à une étroite zone située sous le métalimnion.

Un autre phénomène singulier se manifeste en novembre dans le canal pour Bosmina longirostris, Filinia longiseta, Polyarthra vulgaris et Brachionus calyciflorus. On enregistre en plus des deux maxima de surface, caractéristiques des «twilight migrations»un troisième pic assez aberrant, et bien marqué chez ces quatre espèces. Le pic nocturne excédentaire ne peut en aucun cas être attribué à l'influence de la lumière lunaire (les prélèvements étant effectués à la nouvelle lune). Notons que Kikuchi (1930 a et b) avait observé un phénomène semblable pour l'espèce Bosminopsis deitersi.

\section{Cas particuliers de Keratella quadrata et des nauplii.}

Ce phénomène également singulier des migrations inversées (nocturne pour Keratella quadrata, diurne pour les nauplii) a été 
constaté pour différents lacs et plans d'eau peu profonds : Nichols (1934), Maloney et Tresler (1942) pour des Copépodes, Pennak (1944), George et al. (1969) pour des Rotifères et notamment pour les genres Filinia, Keratella, Polyarthra, capables de présenter une migration normale durant la saison chaude et des migrations inversées en hiver ou au printemps.

Il faut considérer que pour les Copépodes, ces migrations inversées sont ici le fait des plus jeunes stades et que les observations ont eu lieu à l'approche de l'hiver alors que la durée et l'intensité de l'ensoleillement atteignent leurs plus faibles valeurs. Or, selon Clarke (1932) et Cushing (1951), les jeunes seraient souvent beaucoup moins sensibles à la lumière que les derniers stades. Par suite, ils présenteraient plus fréquemment un phototropisme positif. Outre l'âge des animaux et la plus faible intensité des radiations lumineuses en novembre, les basses températures de l'eau durant les récoltes sont également susceptibles d'avoir favorisé ces migrations inversées. Cushing relate l'étude expérimentale entreprise par Dice et Loeb sur des Cladocères : ils constatèrent que des Daphnies présentant un phototropisme négatif à $20^{\circ} \mathrm{C}$ (température estivale) étaient au contraire attirées par la lumière, lorsque la température s'abaissait à $12{ }^{\circ} \mathrm{C}$.

\section{Mouvements horizontaux.}

La surabondance des organismes vers 10 heures tant en surface qu'en profondeur ne peut résulter que d'un pénomène de migration horizontale. Cet enrichissement matinal de la zone médiane du canal pourrait s'expliquer par le fait que la section du plan d'eau n'a pas la forme en auge typique des canaux. En effet, par suite des fréquents éboulements des berges, le fond s'élève assez progressivement du chenal médian vers le littoral. Le mouvement descendant de la fin de la nuit tend à concentrer les organismes vers ce chenal. On peut supposer en effet que près des berges, les organismes qui ont atteint rapidement le fond, continuent leur descente en longeant cette pente douce lorsque le mouvement se renverse. Il en résulte une plus forte concentration du plancton dans le chenal.

\section{Influence éventuelle des paramètres physicochimiques mesurés.}

Le refroidissement et la raréfaction progressive du taux de l'oxygène au cours de la nuit pourraient favoriser une réaction du plancton allant dans le sens d'un géotropisme négatif (Kikuchi 1930). Cependant, étant donné que la majorité des espèces présentes n'ont pas un, mais deux mouvements ascendants nocturnes, il est difficile d'établir une corrélation entre ces processus et les variations de teneur en oxygène ou de température. 
Il ne semble pas que les très faibles variations enregistrées pour le $\mathrm{pH}$, la température ou l'oxygène dissous aient eu une influence décisive sur les mouvements du plancton.

\section{CONCLUSION}

A l'exception des espèces rotifériennes Keratella cochlearis et Brachionus calyciflorus, qui présentet une distribution nocturne quasi normale, on constate qu'en absence de toute turbulence atmosphérique, la distribution des organismes demeure très hétérogène durant la totalité du cycle nycthéméral. Les organismes sont répartis soit selon un gradient de densité allant de la surface vers le fond (Rotifères), soit selon un gradient à partir des couches médianes avec des maxima en surface et en profondeur (Bosmina longirostris).

Pour cette période de récoltes favorisée par des conditions atmosphériques optimales (ciel totalement dégagé, pas de vent) ou presque (luminosités d'intensité plus faible qu'en saison chaude), on peut conclure à l'existence indéniable de migrations verticales. Celles-ci présentent en regard de la faible profondeur du biotope une assez grande amplitude et touchent une fraction non négligeable de la population.

A l'exception des nauplii, les migrations observées pour la plupart des espèces, s'apparente au type des «Twilight migrations» décrites par Cushing (1951) puis Siebeck (1960). On constate cependant à la fin de la nuit un mouvement ascendant supplémentaire, mouvement qui culmine avant l'aurore et qui est plus généralement caractéristique des cas de migrations "nocturnes 》 pour lesquelles les organismes ne migrent qu'une seule fois vers la surface au cours du cycle nycthéméral.

Il va sans dire que l'étude préliminaire des mouvements verticaux du plancton dans ce biotope demande à être complétée par des observations effectuées dans des conditions différentes de luminosité et de température. Toutefois, du point de vue pratique des récoltes, on peut conclure que pour ce biotope et la saison considérée, c'est entre 22 heures et 2 heures du matin que la distribution des organismes est la moins hétérogène. Ce qui nous mène sensiblement aux mêmes conclusions que Hall et al. (1970) et Pourriot (1972) qui constataient respectivement pour divers plans d'eau peu profonds, que la période la plus favorable aux récoltes se situait vers 22 ou 23 heures.

Ces données relatives aux mouvements du plancton sont à la fois déroutantes et contradictoires. En fait si la luminosité constitue un facteur très important, elle intervient en synergie avec différents 
autres paramètres biotiques et abiotiques auxquels les individus sont plus ou moins sensibles selon l'âge ou la saison. Comme le rappelait Hutchinson $(\mathbf{1 9 6 7})$ «... ces types de mouvements sont nombreux et dépendent non seulement de l'espèce, de la localité, mais encore de l'âge et du sexe des individus ».

Ces mouvements permettent non seulement aux espèces animales de varier leurs aires d'activité et de prédation, mais peut-être encore de modifier leur distribution en fonction de celle des autres espèces. Il est en effet curieux de noter qu'au cours des 24 heures, les densités maximales des espèces dominantes n'apparaissent généralement pas dans les mêmes strates de la colonne d'eau comme le constataient Berzin (1968) pour la distribution de 5 espèces de Polyarthra ou George et al. (1970) pour trois autres espèces rotifériennes dominantes. Dans le canal, nous avons pu constater que les aires de répartition de Filinia longiseta, Polyarthra vulgaris, Keratella quadrata et des nauplii de Cyclopidae qui présentent des affinités du point de vue du spectre alimentaire, varient dans l'espace et dans le temps mais se chevauchent assez peu. En atténuant les phénomènes d'exclusion compétitive, ceci doit favoriser la coexistence de ces espèces au sein d'un même plan d'eau.

Il n'est pas impossible que la composition spécifique du plancton puisse avoir des répercussions sur le comportement migratoire de chaque espèce, au même titre que l'âge ou le sexe des individus.

\section{TRAVAUX CITÉS}

Angeli (N.). 1972. - Esquisse du profil biologique (plancton) d'un canal influencé par une pollution complexe d'origine industrielle : le canal de Seclin-Nord. Biol. Jb. Dodonaea, $40: 77-115$.

Cuarke (G. L.). 1932. - Quantitative aspects of the change of phototropic sign in Diaphnia. J. Exp. Biol., $9: 180-211$.

Cushing (D. H.). 1951. - The vertical migration of plankton crustacea. Biol. Rev, $26: 158-192$.

George (M. G.). 1961. - Diurnal migrations in two shallow ponds in Delhi, India. Hydrobiol., 18 (3) : 265-273.

George (M. G.) et Fernando (C. H.). 1969. - Seasonal distribution and vertical migration of planktonic Rotifers in two lakes in eastern Canada. Verh. Internat. Verein. Limnol., 17 : 817-829.

George (M. G.) et Fernando (C. H.). 1970. - Diurnal migrations in three species of Rotifers in sunfish lake Ontario. Limnol. Oceanogr., 15 (2) : 218-223.

Hall (D. J.), Cooper (W. E.) et Werner (E. E.). 1970. - An experimental approach to the production dynamics and structure of freshwater animals communities. Limnol. Oceanogr., 15 (6) : 839-928.

Hutchinson (G. E.). 1967. - A treatise of Limnology. Vol. II. Introduction to lake biology and the limnoplankton, $1115 \mathrm{pp}$.

KIKUCHI (K.). 1930 a. - A comparison of the diurnal migration of plankton in eight Japanese lakes. Mem. Coll. Sci. Kyoto Univ., ser. B (5) : 27-46. 
Kikuchi (K.). 1930 b. - Diurnal migration of Plankton Crustacea. $Q$. Rev. Biol., 5 : 189-206.

LARSSON (P.). 1970. - Vertical distribution of Plankton Rotifers in a meromictic lake, Blankvant near Oslo. Norw. J. Zool., 19 (I) : 47-75.

Maloney (M. T.) et Tresier (W. L.). 1942. - The diurnal migration of certain species of zooplankton in Caroga lake, New York. Trans. Am. Microsc. Soc., 61 : 40-52.

Nicholss (A. G.). 1934, - On the biology of Calanus finmarchicus. III. Vertical distribution and diurnal migration in the Clyde sea area. J. Mar. Biol. Ass. U. K., 19139.

Omaly (N.). 1970. - Première contribution à l'étude des bassins de la Haute Tarentaine. Le lac de la Cregut et ses annexes : évolution, microendémisme chez les Calanides. Ann. Stat. Biol, Besse-enChandesse, 5 : $303-347$.

Pawłowski (L. K.). 1970. - Les Rotifères de la rivières Grabia : variations observées au cours d'un cycle de 24 heures dans la composition de la faune des Rotifères. Pracewydz. III Nauk. Mat. - Przyr. Lodz, $110: 1-127$.

PenNaK (R. W.). 1944. - Diurnal movements of zooplankton organisms in some Colorado mountains lakes. Ecol., 25 : 387-403.

Pourriot (R.). 1970. - Etude hydrobiologique de deux petits étangs de prairie. Observations sur la distribution de la température et du plancton et l'influence d'un couvert végétal Lemna minor. Ann. Hydrobiol., 3 (I) : 33-46.

Schindler (D. W.), Noven (B.). 1971. - Vertical distribution and seasonal abundance of zooplankton in two shallow lakes of the experimental area, Northen Ontario. J. Fish. Res. Bd. Canada, 28 (2) : 245-256.

Schröber (R.). 1959. - Die Vertikalwanderungen der Crustaceenplanktons der Seen des Südlischen Schwarzwaldes. Arch. Hydrobiol., suppl. $25: 1-43$.

Siebeck (O.). 1960. - Untersuchungen über die vertikal Wanderung planktischer Crustaceen unter besonderer Berücksichtingung der Stralungsverhältnisse. Int. Revue ges. Hydrobiol. Hydrogr., 45 : 381-454.

Si.A $\AA_{\text {Deček }}$ (V.), Fiala (L.) et Sladeckova (A.). 1959. — Limnologische Forschungen am Stausee Pastviny mit besonderer Berücksichtigung des Einflusses eines Kraftwerkes. Pap. Inst. Chem. Techno.l, Fac. Technol. Fuel and Water, 3 (2) : 431-595.

Worthington (E. B.). 1931. - Vertical movements of freshwater macroplankton. Int. Revue ges. Hydrobiol. Hydrogr., 25 : 394-436. 